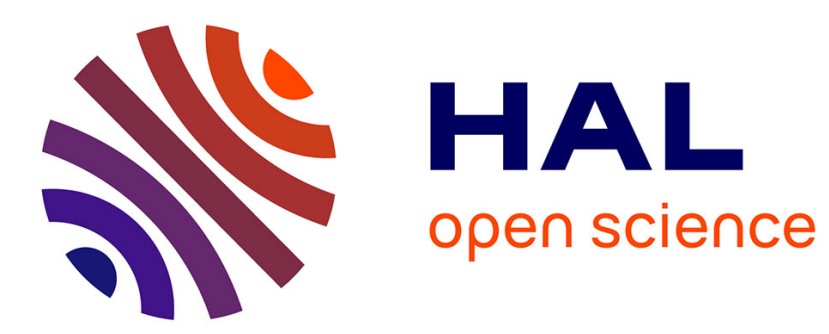

\title{
Performance of a Small MWPC Based Pet System
}

M. Staric, D. Korbar, A. Stanovnik

\section{To cite this version:}

M. Staric, D. Korbar, A. Stanovnik. Performance of a Small MWPC Based Pet System. Journal de Physique IV Proceedings, 1995, 05 (C1), pp.C1-207-C1-214. 10.1051/jp4:1995124 . jpa-00253560

\section{HAL Id: jpa-00253560 https://hal.science/jpa-00253560}

Submitted on 1 Jan 1995

HAL is a multi-disciplinary open access archive for the deposit and dissemination of scientific research documents, whether they are published or not. The documents may come from teaching and research institutions in France or abroad, or from public or private research centers.
L'archive ouverte pluridisciplinaire HAL, est destinée au dépôt et à la diffusion de documents scientifiques de niveau recherche, publiés ou non, émanant des établissements d'enseignement et de recherche français ou étrangers, des laboratoires publics ou privés. 


\title{
Performance of a Small MWPC Based Pet System
}

\author{
M. Starič, D. Korbar and A. Stanovnik* \\ I. Stefan Institute and Physics Department, University of Ljubljana \\ * Faculty for electrical engineering, University of Ljubljana, Ljubljana, Slovenia
}

\begin{abstract}
In our rescarch, we are attempting to construct a PET apparatus with superior position resolution in comparison with commercially available systems. Tests of a small apparatus $\left(5 \times 5 \mathrm{~cm}^{2}\right)$, hased on multiwire proportional chambers, have shown a position resolution of $3 \mathrm{~mm}$ fiul width at half maximum (FWIIM) in all three dimensions of the reconstructed tomographic image of a point source. The other parameters of the system are: 8 ns FWHM timing resolution and $0.5 .5 \%$ efficiency for one chamber layer. Based on these results the construction of a larger system $\left(32 \times 32 \mathrm{~cm}^{2}\right)$ with eight chamber layers (efficiency $=4 \%$ ) is in progress.
\end{abstract}

\section{INTRODUCTION}

The position resolution of positron emission tomography (PET) is limited by the range of positrons and by the noncollinearity of the two anihilation photons due to the $e^{+} e^{-}$center-of-mass motion. For imaging of living systems, these two effects contribute about, $2 \mathrm{~mm}$ to the full width at half maximum (FWHM). In order to acheive such a resolution of the reconstructed image, the detectors of the PET system should be capable of localizing the anibilation photon hits with a better or at least comparable accuracy.

It seems that multiwire proportional chambers (MWP('s) offer this possibility [1-1]. In addition, the large surface of these detectors enables imaging of larger volumes. The main disadvantage of MWP('s for PET is in their low efficiency for detection of $511 \mathrm{keV}$ photons. For some applications in medical, biological or physics research, it might be worth trading the good efficiency of commercial, scintillator-based systems for the better position resolution, large surface and low cost of PET systems based on multiwire proportional chambers.

In this work we present the results of our attempts to improve some of the parameters of a MWPC based PET system. For this purpose, we have constructed small $\left(50 \times 50 \mathrm{~mm}^{2}\right)$ wire chambers with $\mathrm{Pb}-\mathrm{Bi}$ cathode strips as converters and with delay line readout of the coordinates.

\section{CALCULATIONS AND ESTIMATES OF PARAMETERS}

Monte Carlo simulations of the distribution of events with respect to the range of positrons in water (which is aproximately equal to that in tissue) are shown in Fig. 1 for the most commonly used positron emitters. The finite value of the $\mathrm{dN} / \mathrm{dr}$ distribution at zero range corresponds to a singularity in the density of events $\mathrm{dN} / \mathrm{dV}$. It is thus not accurate to consider the positron range as an independent contribution to the width of a point source image. Inclusion of $\gamma-\gamma$ noncollinearity (10 mrad FWHM and a point source in the center of an ideal spherical cletector of $2 \mathrm{r}=58 \mathrm{~cm}$ ), 


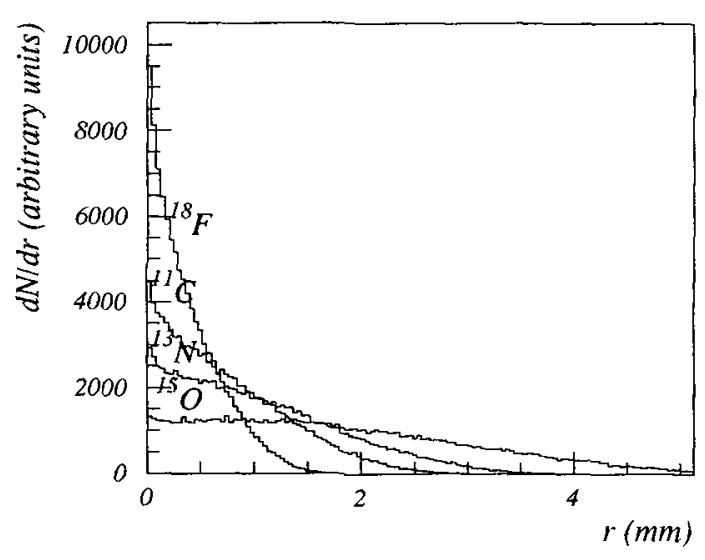

FIG.1: Distribution of the positron range for some commonly used positron emitters.

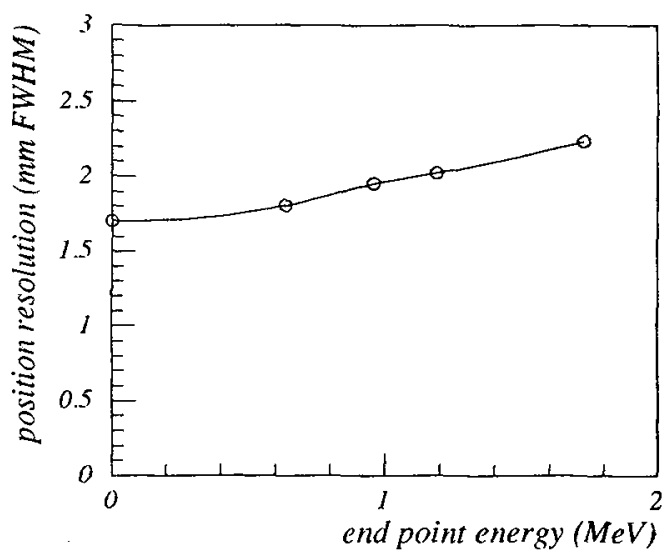

FIC.2: Dependence of PET resolution on the end-point energy of the $\beta$-spectrum if only positron range and $\gamma-\gamma$ noncollinearity are taken into account. The detector is spherical with $2 \mathrm{r}=58 \mathrm{~cm}$ and perfect resolution.

shows that the width of a point source image depends only weakly on the $\beta$-spectrum end point energy (Fig.2), although the average range varies considerably $\left(0.4 \mathrm{~mm}\right.$ for ${ }^{18} \mathrm{~F}$ to $1.8 \mathrm{~mm}$ for $\left.{ }^{15} \mathrm{O}\right)$. Higher end point energies mainly cause high "wings" of the distribution and thus influence the image contrast as may be seen in Fig.3.

The position resolution of a MPC is given by the difference between the conversion point and the measured avalanche position. Assuming that electrons emerge isotropically from the cathode-strip converter of a symmetric MWPC, the distribution of these differences would be: $\frac{d N}{d x}=\frac{N}{\pi L} \frac{1}{1+(x / L)^{2}}$, where L is the anode-to-cathode separation [5]. The FWHM of this distribution is equal to the chamber thickness $2 \mathrm{~L}$. The convolution for two detertors gives a position resolution of $2 \mathrm{~L} / \sqrt{2}$ at the midplane between them. Summing up the squares of contributions to position resolution, one sees that a chamber thickness of about $2 \mathrm{~mm}$ would increase the position resolution by $25 \%$ over the ireducible contribution from positron range and $\gamma-\gamma$ noncollinearity.

Measurement of coincident events in two MWPC's requires a certain time window, which depends on the time resolution of both detectors. In a MWP( , the time resolution is determined mainly by the drift of electrons towards the anode wire and the FWHM is estimated at $\delta t=s / 4 w_{d}$, where $s$ is the anode wire pitch and $w_{d}$ the electron drift velocity. For a $5 \mathrm{kV} / \mathrm{cm}$ electric field in the MWPC, the drift velocity in NTP methane is about $50 \mathrm{~mm} / \mathrm{ms}, \mathrm{sos}=2 \mathrm{~mm}$ gives a time resolution of about $10 \mathrm{~ns}$.

In designing the MWP(, we have chosen standard printed circuit boards (PC,B) of $1.55 \mathrm{~mm}$ thickness $(\mathrm{L}=1.55 \mathrm{~mm})$ accurate to about $10 \mu \mathrm{m}$ rms. The anode wires were of gold-plated tungsten with $2 \mathrm{r}=10 \mu \mathrm{m}(1 \pm 0.01)$. Assuming that the wire positioning is accurate to $\pm 50 \mu \mathrm{m}$ $\mathrm{rms}$, we performed calculations of the multiplication factor for three values of the anode wire pitch $(\mathrm{s}=2 \mathrm{~mm}, 1 \mathrm{~mm}$ and $0.5 \mathrm{~mm})$. With all the parameters distributed around their mean values according to Gaussian distributions, we obtain distributions of the multiplication factor which are not symmetric and have standard deviations of $8 \%, 22 \%$ and $51 \%$ in the three cases respectively. These results indicate that $2 \mathrm{~mm}$ anode wire pitch guarantees stable operation at the highest average multiplication and acceptable wire positioning tolerance of $50 \mu \mathrm{m}$.

For MWPC based PET systems, the converters for $511 \mathrm{keV}$ photons determine the system efficiency, the time resolution and the position resolution [1-5]. Our choice of cathode plane converters, 


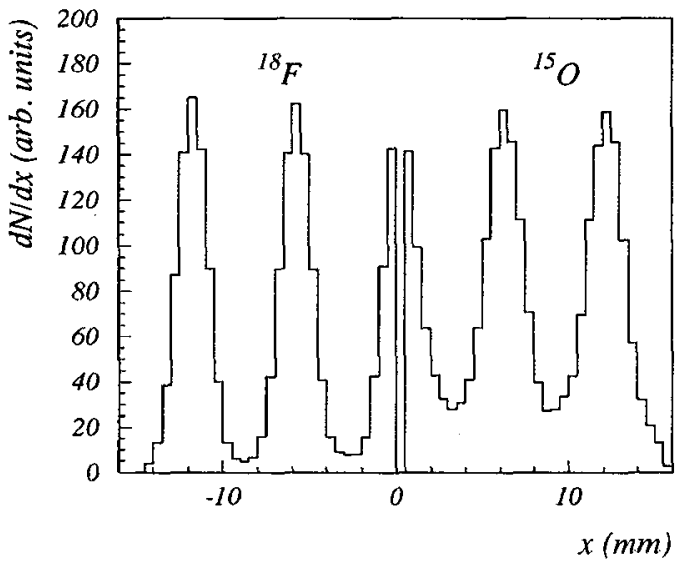

FIG.3: Image reconstruction of simulated ${ }^{18} \mathrm{~F}$ and ${ }^{15} \mathrm{O}$ point sources. Notice that the higher end-point energy of ${ }^{15} \mathrm{O}$ mainly increases the background.

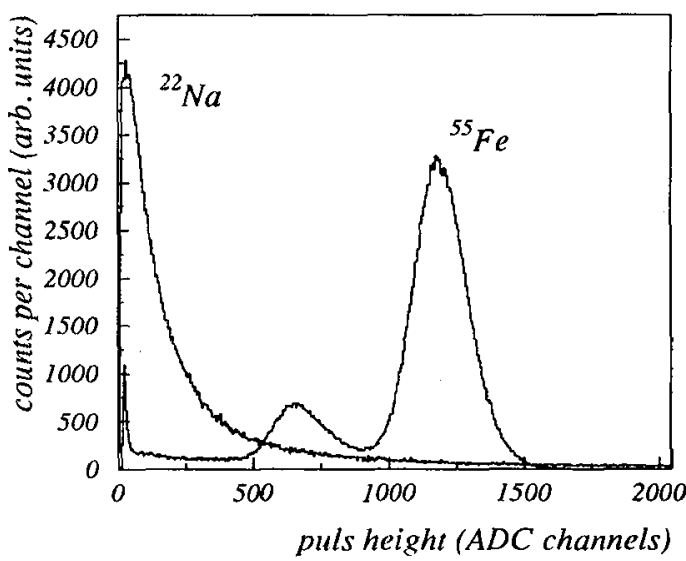

FIG.4: MWPC pulse height distributions due to ${ }^{55} \mathrm{Fe}(5.9 \mathrm{keV})$ and ${ }^{22} \mathrm{Na}(511 \mathrm{keV})$ sources. The full energy peak and the $K_{\alpha}$ escape peaks are seen for ${ }^{55} \mathrm{Fe}$, whereas the $51 \mathrm{keV}$ photons are detected via recoil electrons escaping out of the converter.

with the Cu coating on the PCB's divided into strips $(1.5 \mathrm{~mm}$ wide, $0.5 \mathrm{~mm}$ gaps), which are covered with $\mathrm{Pb}-\mathrm{Bi}(0.1 \mathrm{~mm}$ average thickness with the shape determined by surface tension), has the advantage of a simple production procedure while maintaining good values of the parameters. Measurements of these parameters are presented in the next section.

\section{MEASUREMENTS}

\subsection{Pulse height distributions}

A MWPC of $50 \times 50 \mathrm{~mm}^{2}$ active area, with $2 \mathrm{~mm}$ anode wire pitch, $1.55 \mathrm{~mm}$ anode-to-cathode spacing, one cathode plane consisting of $\mathrm{Pb}$ - $\mathrm{Bi}$ strips, the other being a wire plane, has been assembled and tested with P10 $\left(90 \% \mathrm{Ar}+10 \% \mathrm{CH}_{4}\right)$ as the chamber gas. The spectrum obtained by irradiating the MWPC from the wire-cathode side, with a ${ }^{55} \mathrm{Fe}$ source (5.9 keV X-rays) is shown in Fig.3, together with the pulse height distribution caused by conversion of $511 \mathrm{keV}$ photons, obtained by irradiation of the MWPC from the $\mathrm{Pb}-\mathrm{Bi}$ converter side with a ${ }^{22} \mathrm{Na}$ source. The $5.9 \mathrm{keV}$ peak corresponds to 210 ion pairs, which gives 40 ion pairs for the average pulse height caused by conversion of $511 \mathrm{keV}$ photons. The limit of proportionality is at $1.10 \mathrm{kV}\left(M=2 \times 10^{4}\right)$ and the limit of stability at $1.28 \mathrm{kV}\left(M=2 \times 10^{5}\right)$. Measurements of the $5.9 \mathrm{keV}$ peak with a collimated ${ }^{55} \mathrm{Fe}$ source show that the central $42 \times 45 \mathrm{~mm}^{2}$ surface of the MWPC has uniform response within $10 \%$.

\subsection{Position resolution}

The cathode PC boards have been designed so that the strips could be capacitively coupled to delay lines on the outer side of the chambers and that this coupling compensated the dispersion of signal propagation along the delay line [5]. A charge sensitive preamplifier at each end of the delay line, amplified and adjusted the signals, which were then led to STOP inputs of a TDC CAMAC module (Silena). The START was provided by the anode plane signal. The distribution of time differences of signals from both ends of the delay line for $x$ and $y$ cathode strips, when the chamber is iradiated uniformly over it's surface, is shown in Fig.5. The wire structure seen in the y direction permits calibration of position versus TDC channels as the wire positions are known to an accuracy 

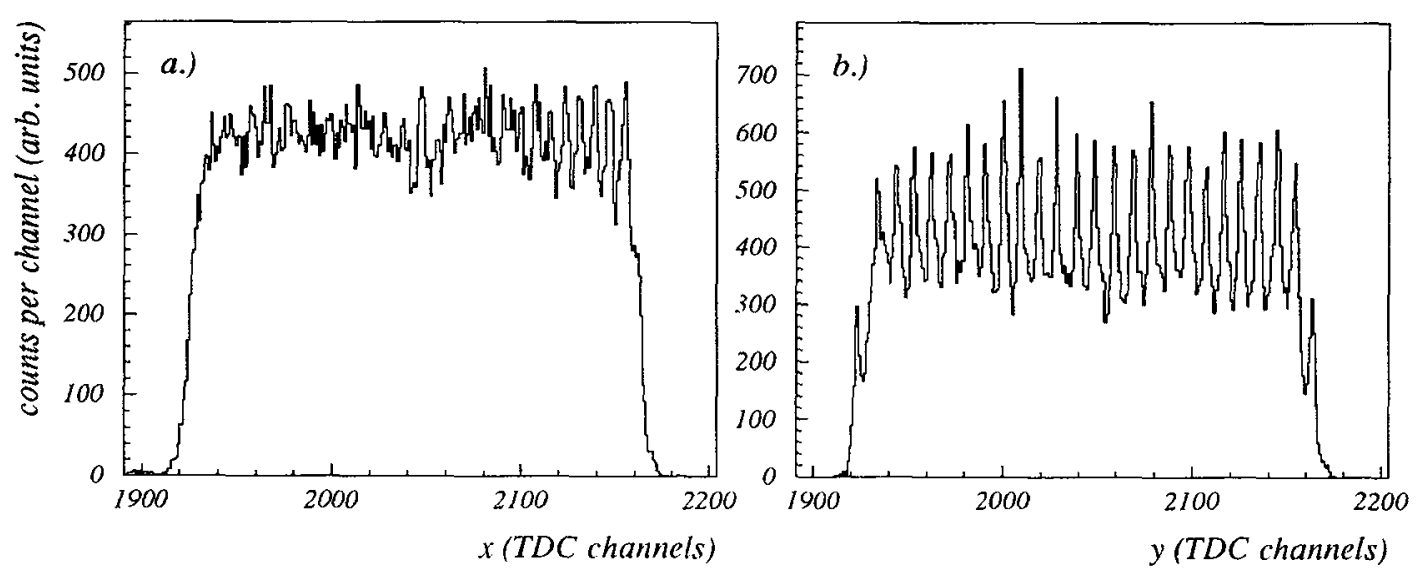

FIG.5: Distribution of time differences of pulses at both ends of delay lines when the MWPC is uniformly irradiated.

a) Cathode strips are orthogonal to the anode wires (x-coordinate).

b) Cathode strips are parallel to the anode wires (y-coordinate).

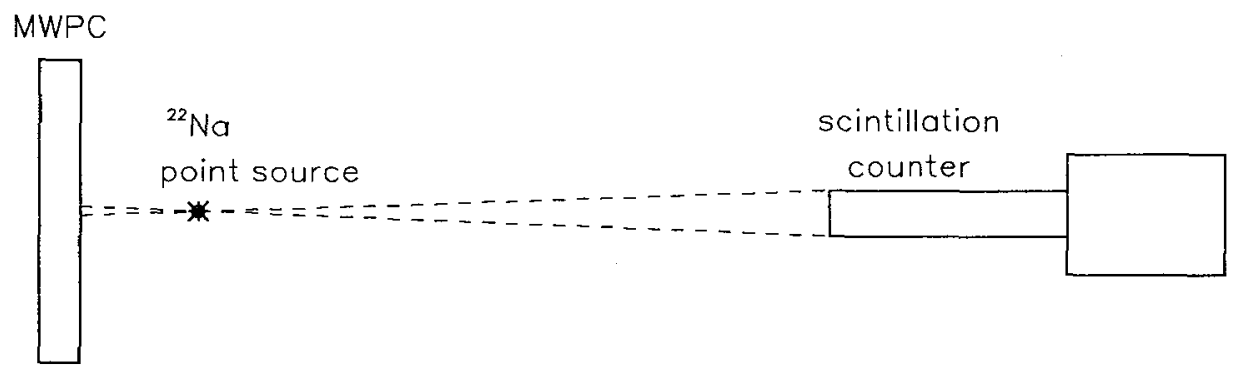

FIG.6: Set-up for measuring position resolution of the MWPC: by coincidence of collinear anihilation photons. The ${ }^{22} N a$ source image on the MWPC is $1 \mathrm{~mm}$ wide. 

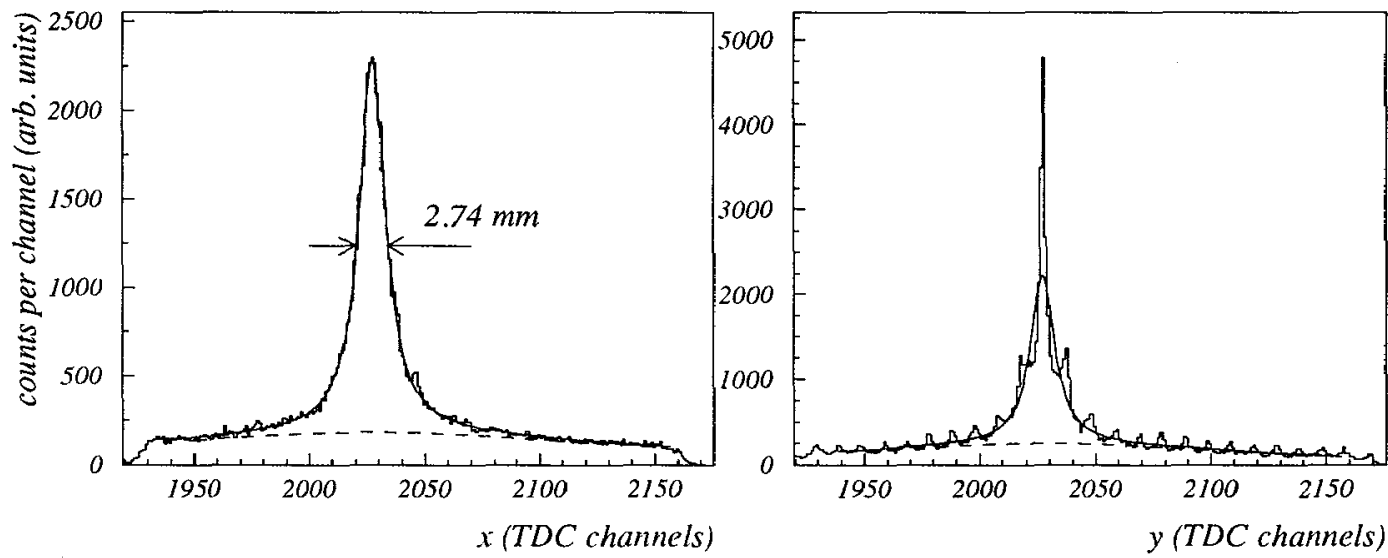

FIG.7: Position resolution of the MWP(, for cathode strips orthogonal to the anode wires ( $\mathrm{x}$ coordinate) and for cathode strips parallel to the anode wires ( $y$-coordinate). The peak is due to coincidences of both $511 \mathrm{keV}$ anihilation photons (see Fig.6), while the background (dashed line) comes from coincidences of one $1275 \mathrm{keV}$ and one $511 \mathrm{keV}$ photon. (1 TDC channel = $200 \mu \mathrm{m})$

of $50 \mu \mathrm{m}$.

The MWPC position resolution has been obtained by measuring collinear $511 \mathrm{keV}$ anihilation photons from a ${ }^{22} \mathrm{Na}$ point source in coincidence with a scintillation counter (Fig.6). The dimensions of the scintillator image on the chamber were $1 \mathrm{~mm} \times 10 \mathrm{~mm}$. For this measurement the MWPC detector consisted of four chamber layers (1 layer $=1$ anode wire plane +1 cathode plane with horizontal $\mathrm{Pb}-\mathrm{Bi}$ strips +1 cathode plane with vertical $\mathrm{Pb}$-Bi strips). The measured distributions of Fig.7 have been fitted with functions of the form given in section 2. For the first and the third chamber layer, where anode wires are orthogonal to the scintillator image, we obtain $3.07 \mathrm{~mm} \pm 0.05 \mathrm{~mm}$ and $2.74 \mathrm{~mm} \pm 0.05 \mathrm{~mm}$ for the full width at half maximum. Subtracting in quadrature the beam width of $1 \mathrm{~mm}$, we obtain $2.8 \mathrm{~mm}$ and $2.6 \mathrm{~mm}$. This is in quite good agreement with our prediction in section 2, where we stated that the FWHM is equal to the chamber thickness. Namely, if we subtract the converter maximum thickness of $2 \times 0.2 \mathrm{~mm}$ (one on each cathode) from the cathode-to-cathode distance $(2 \times 1.55 \mathrm{~mm}=3.1 \mathrm{~mm})$ we obtain $2.7 \mathrm{~mm}$.

\subsection{Time resolution}

The time resolution was measured with the same set-up as shown in the previous subsection. Using constant fraction discrimination, the time resolution of the scintillation counter is below 1 ns and could be neglected in comparison with the MWPC time resolution. With methane at NTP as the chamber gas and a voltage of $1.5 \mathrm{kV}$, we measured the distribution shown in Fig.8. At higher voltages the width increases (to $8.1 \mathrm{~ns}$ FWHM at $1.8 \mathrm{kV}$, see Fig.8) as the drift velocity in methane decreases with increasing electric field [6]. 

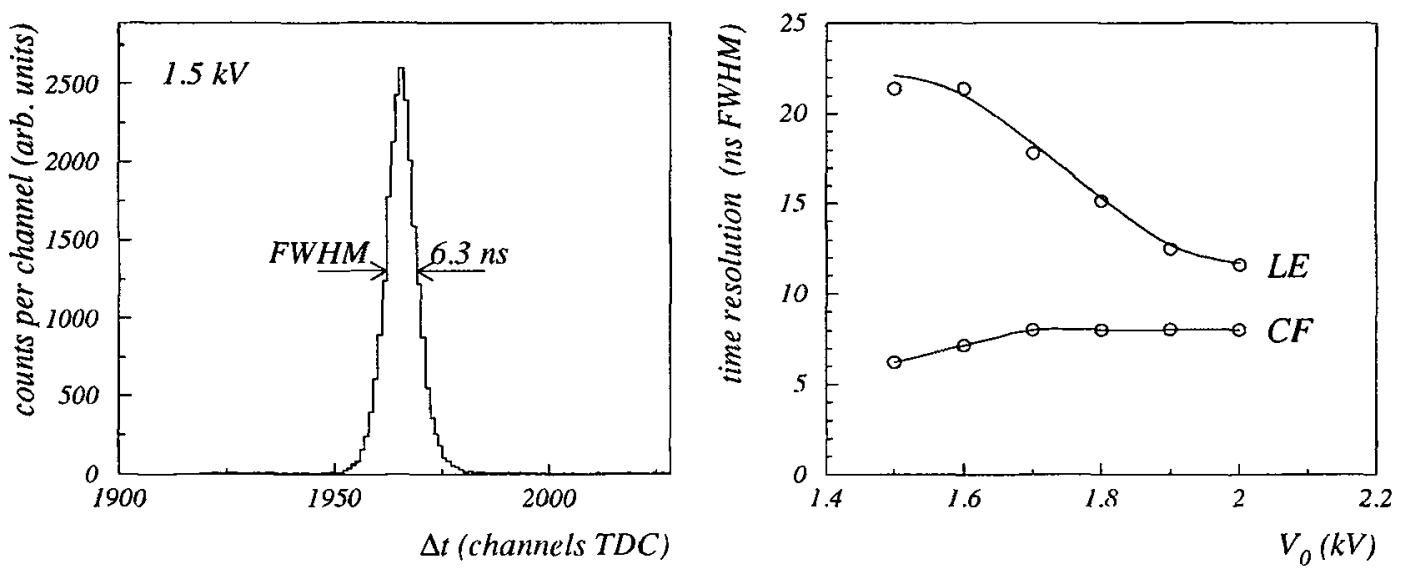

FIG.8: On the left is the distribution of time differences between the MWPC and scintillation counter signals for coincident anihilation photons. On the right is the dependence of time resolution on anode high voltage with methane gas filling for leading edge and constant fraction discrimination.

\subsection{Efficiency}

Using data for fotoeffect and Compton scattering cross sections from the literature $[7,8]$ as well as transmission of various materials for electrons $[9,10]$, we have calculated the fraction of conversion electrons escaping from the converter in the direction of the incident photon [5]. Fig.9 shows this calculated efficiency (number of escaped converted electrons per incident photon) for $150 \mathrm{mg} / \mathrm{cm}^{2}$ thick converters as a function of the atomic number $\mathrm{Z}$.

The MWPC efficiency has been measured by the coincidence rate of signals due to $511 \mathrm{keV}$ photons being absorbed in the MWPC and in a NaI scintillation counter. As this coincidence rate depends on the product of efficiencies of both detectors, the $\mathrm{NaI}$ counter efficiency had been obtained by Monte Carlo simulations. A separate measurement, with the $\mathrm{NaI}$ counter rotated out of the solid angle determined by the ${ }^{22} \mathrm{Na}$ point source and the MWPC, was made in order to subtract coincidences of noncollinear $511 \mathrm{keV}$ and $1275 \mathrm{keV}$ photons.

Efficiency measurements for converters of various atomic numbers $\mathrm{Z}$ (vetronite + thin graphite layer, vetronite $+30 \mu m \mathrm{Cu}, \mathrm{Sn}-\mathrm{Pb}$ (solder layer), $50 \mu m \mathrm{~Pb}, 1.25 \mathrm{~mm} \mathrm{~Pb}$ and $100 \mu \mathrm{m} \mathrm{Pb}-\mathrm{Bi}$ ) are compared with the calculated values in Fig.9. For this purpose, one of the cathodes was a wire plane with negligible efficiency, so the converter cathode efficiency could be measured with $511 \mathrm{keV}$ photons incident from either side. For the $\mathrm{Pb}-\mathrm{Bi}$ cathode strips of $0.1 \mathrm{~mm}$ average thickness, the measured efficiency is $0.37 \%$, when the electron escapes in to the chamber along the direction of the incident photon and $0.18 \%$, when the electron is backscattered in the converter. The efficiency of one chamber layer is thus $0.55 \%$. With a detector consisting of four chamber layers, the measured efficiency is $1.9 \%$. The energy dependence of the efficiency for different numbers of MWPC layers with $\mathrm{Pb}-\mathrm{Bi}$ cathode strip converters is shown in Fig. 10. 


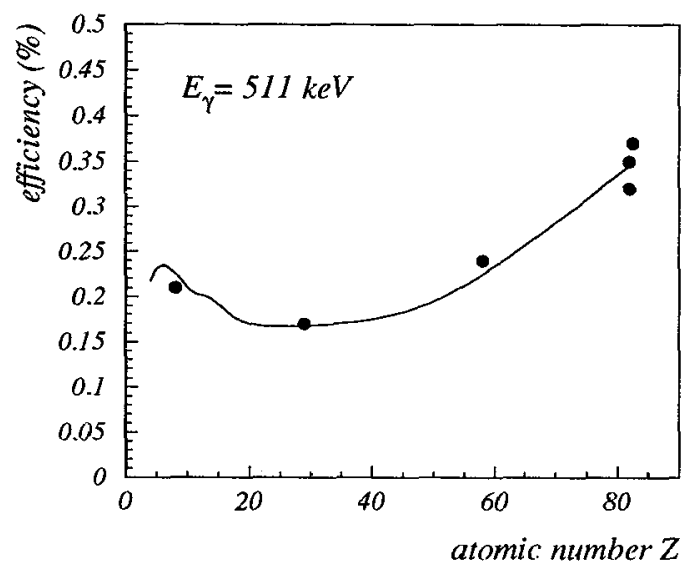

FIG.9: Calculated (full line) and measured (black dots) efficiencies for for $511 \mathrm{keV}$ photon conversion and electron escape in 150 $\mathrm{mg} / \mathrm{cm}^{2}$ thick absorbers as a function of the average atomic number of the converter.

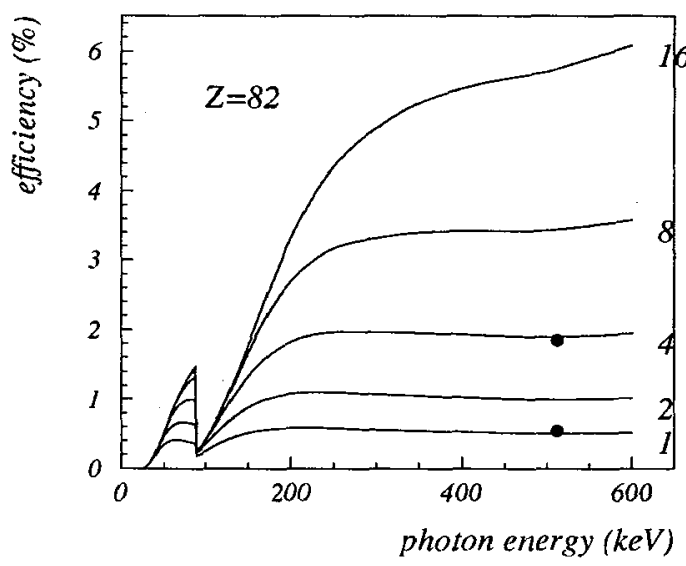

FIG.10: Energy dependence of the efficiency for MWPC detectors with different numbers of chamber layers (one chamber layer has two $\mathrm{Pb}$-Bi cathode plane converters). Black dots are measured values.

\section{3D IMAGING OF POINT SOURCES}

Finally, two chambers have been used to measure anihilation photons from two ${ }^{22} \mathrm{Na}$ point sources. The reconstruction algorithms require an axially symmetric measurement so the sources have been mounted on a computer controlled turntable and rotated with a stepping motor (200 steps/turn). The 200000 measured events have been backprojected into a $(32 \mathrm{~mm})^{3}$ volume, divided into $64^{3}$ voxels, which required 3.5 minutes on a $\mu V A X I V$ computer. After filtering with a Gaussian $(\sigma=1$ voxel), the 3D distribution has been obtained by deconvolution with an analytical expression for the point source response function (PSRF)[11]. This required 26 seconds on the $\mu V A X I V$. Slices and cuts through the reconstructed image of both point sources show a full width at half maximum of 3 $\mathrm{mm}$ (Fig.11). We recall that this number includes positron range, $\gamma-\gamma$ noncollinearity and position resolution of both chambers.

\section{CONCLUSION}

The performance of thin wire chambers with $1.55 \mathrm{~mm}$ separation between anode and cathode, with $2 \mathrm{~mm}$ anode wire pitch and with the cathode strips of both cathode planes covered with a $\mathrm{Pb}-\mathrm{Bi}$ layer has been tested. The position resolution of the reconstructed image of a point source is $3 \mathrm{~mm}$ FWHM, the time resolution of a chamber is $8 \mathrm{~ns}$ and the efficiency for one chamber layer is 0.55 $\%$. The efficiency could be increased by increasing the number of chamber layers. The measured values of these parameters all agree with predictions based either on straightforward calculations or on Monte Carlo simulations. Besides the superior position resolution it is also the availability of large surfaces and their low cost that make multiwire chambers attractive as detectors in a PET system. We beleive that a larger system $\left(32 \times 32 \mathrm{~cm}^{2}\right.$ presently under contruction $)$ will find usefull applications in biological and medical research. 

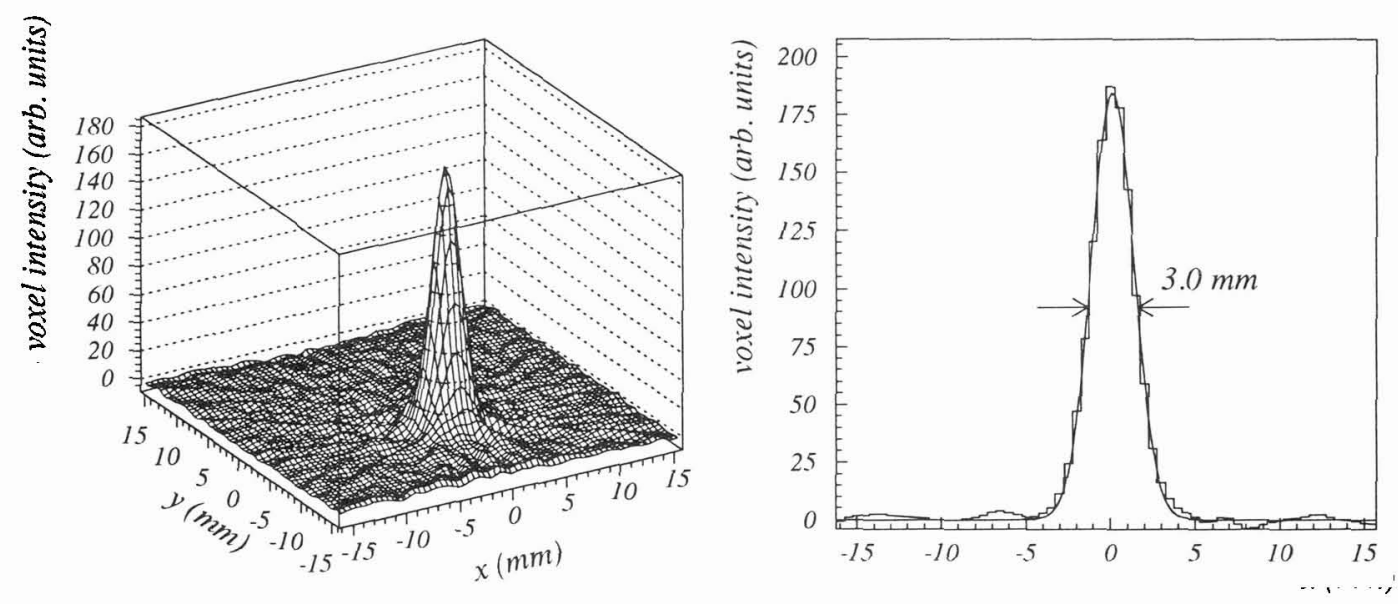

FIG.11: Reconstruction of point-source images. On the left is a slice through one of the two reconstructed point sources. The histogram on the right shows the distribution through a point source image in the $\mathrm{x}$-direction. The other two directions also show a FWHM of $3 \mathrm{~mm}$.

\section{References:}

1. G.Charpak, Applications of proportional chambers to some problems in medicine and biology, Nucl. Instr. Meth. 156(1978)1

2. D.Townsend, P.Frey, A.Jeavons, G.Reich, H.J.Tochon-Danguy, A.Donath, A.Christin and G.Schaller, High Densitiy Avalanche Chamber (HIDAC) Positron Camera, J. Nucl. Med. 28(1987) 1554

3. S.Webb, R.J.Ott, J.E.Bateman, A.C.Flesher, M.A.Fowler, M.O.Leach, P.Marsden, O.Khan and V.R.McCready, Tumour localisation in oncology using positron emitting radiopharmaceuticals and a multiwire proportional chamber positron camera; techniques for $3 \mathrm{D}$ deconvolution, Nucl. Instr. Meth. 221(1984)233

4. A.Del Guerra, A.Bandettini, M.Conti, G.De Pascalis, P.Maiano, V.Perez-Mendez and C.Rizzo, Test of the HISPET Prototype, presented at the IEEE Symposium on Nuclear Science, San Francisco, January 1990

5. M.Starič, PhD thesis, University of Ljubljana, 1992

6. A.Jeavons, K.Kull, B.Lindberg, G.Lee, D.Townsend, P.Frey, and A.Donath, Nucl. Instr. Meth. 176(1980) 89

7. F.Sauli, CERN Yellow Report 77-09 (1977)

8. Photon Cross Section, Attenuation Coefficients and Energy Absorption Coefficients from $10 \mathrm{keV}$ to $100 \mathrm{GeV}$, NBS 29, 1969

9. H.H.Seliger, Phys. Rev. 100/4(1955)1029

10. L.V.Spencer, Phys. Rev. 98/6(1955)1597

11. D.Korbar, A.Stanovnik and M.Starič, Institute Jožef Stefan report IJS DP-5183 (1988) 